\title{
Primum nocere
}

\author{
Roland Schulze-Röbbecke
}

Bis heute gilt es in der Medizin als Zeichen von Bildung und wissenschaftlicher Autorität, wenn man lateinische Sprüche in den Text eines Fachartikels einstreut. „Primum non nocere“ („zuerst einmal nicht schaden“) ist z. B. solch ein Spruch, der sich in letzter Zeit zunehmender Beliebtheit erfreut (Abb. 1). Über seinen Sinn scheint sich indes kaum jemand im Klaren zu sein.

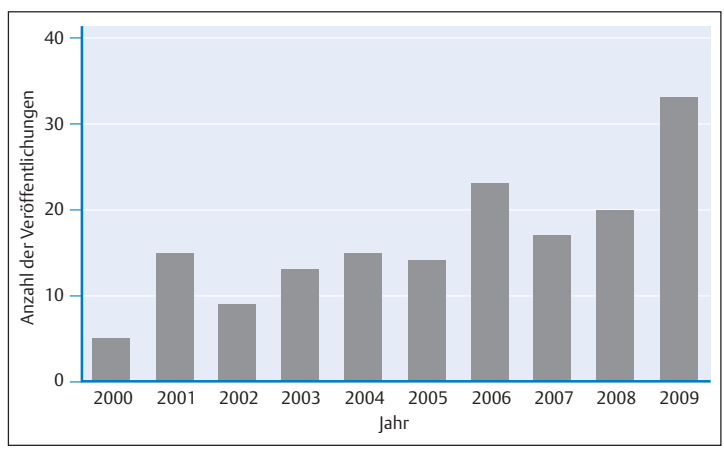

Abb. 1 Anzahl der Veröffentlichungen mit Nennung von „primum non nocere“ im Laufe der letzten 10 Jahre (Daten: PubMed).

Schon die hippokratischen Schriften enthalten Empfehlungen es zu vermeiden, seinen Patienten Schaden zuzufügen. Nirgendwo findet sich dort aber die Aussage, dass dies die erste Pflicht („primum“) des Arztes sei [1]. In der Tat wäre die ärztliche Berufsausübung unmöglich, würde man „primum non nocere“ zur Maxime des ärztlichen Handelns machen. Keine Operation, keine Medikamentengabe und kaum irgendeine andere ärztliche Maßnahme ist frei von potenziell schädigenden Nebenwirkungen; dies gilt selbst für die bloße Anwesenheit eines Arztes [2] und für die Verabreichung von Placebos [3]. Aus ethischer Sicht ist es von Bedeutung, dass der Nutzen einer ärztlichen Maßnahme ihren Schaden überwiegt oder dass die Wahrscheinlichkeit größer ist, dem Patienten mit einer bestimmten Maßnahme zu nützen als ihm damit zu schaden $[1,4,5]$. Nicht „primum non nocere“ darf daher die Maxime des ärztlichen Handelns sein, sondern „primum prodesse“ („zuerst einmal nützen“). Unter Berücksichtigung des Schadens kann man auch den Grundsatz aufstellen „primum prodesse, secundum non nocere“ („zuerst einmal nützen, an zweiter Stelle nicht schaden“).

Wenn nun der Grundsatz „primum non nocere“ für die klinische Medizin unbrauchbar ist, gilt er dann nicht wenigstens für die Präventivmedizin, namentlich für die Infektionsprävention und Hygiene? Dies ist nicht der Fall. Bei der Schutzimpfung setzen wir gesunde Personen dem Risiko schädigender Nebenwirkungen aus im Vertrauen, dass statistisch der Nutzen das Risiko überwiegt. Im Vertrauen auf solch positive Nutzen-Risiko-Bilanzen verabreichen wir eine perioperative Antibiotikaprophylaxe, entziehen wir beatmeten Patienten die Stressulcus-Prophylaxe und desinfizieren wir Oberflächen lacrimae quoad profluunt. Auch hier gilt also das Gebot „primum prodesse, secundum non nocere“. Wie verhält es sich aber mit der Aufbereitung chirurgischer Instrumente oder dem Tragen steriler Schutzkleidung beim Operieren? Gilt hier nicht ausschließlich der Aspekt des Nicht-Schadens, gilt hier nicht uneingeschränkt „primum non nocere“? Nein, denn auch solche Maßnahmen haben sowohl einen Nutzen- als auch einen Schadensaspekt und auch hier sind - wie in der klinischen Medizin - Nutzen und Schaden gegeneinander abzuwägen. Der Nutzen dieser Maßnahmen liegt in der Abwendung von Schäden, z.B. dem Risiko postoperativer Wundinfektionen. Ihr Schaden liegt dagegen im Verbrauch von Ressourcen, die in der Krankenversorgung stets limitiert sind und nach Verbrauch nicht mehr für andere Präventionsmaßnahmen zur Verfügung stehen [6].

Die Relation von Kosten (= Schaden) und Nutzen von Präventionsmaßnahmen wird als „Kosteneffektivität“ bezeichnet. Während sich die Kosten durch einen monetären Betrag charakterisieren lassen, werden zur Darstellung der Nutzeffekte nicht-monetäre, mit Hilfe klinischer Studien ermittelte Indexmaße wie qualitätskorrigierte Lebensjahre (Quality-adjusted life years) herangezogen. Aus gesundheitsökonomischer und gesellschaftlicher Sicht sind präventive Handlungsalternativen mit günstigerer Kosteneffektivität zu bevorzugen [6]. 
Infektionspräventive Maßnahmen können also nicht nur ineffektiv, sie können sogar schädlich sein. Da sie aufgrund des Verbrauchs limitierter Ressourcen miteinander konkurrieren, richtet derjenige einen Schaden für Patienten (ggf. auch für medizinisches Personal) an, der ineffektive Maßnahmen oder Maßnahmen mit ungünstiger Kosteneffektivität bevorzugt und dadurch Maßnahmen mit günstigerer Kosteneffektivität verhindert. Dies soll anhand folgender Beispiele verdeutlicht werden:

- Hygienepersonal schädigt seine Patienten, wenn es seine Arbeitszeit überwiegend mit Umgebungsuntersuchungen oder Fragen der Kleiderordnung zubringt und darüber kosteneffektive Maßnahmen wie die Surveillance nosokomialer Infektionen oder Personalschulungen über evidenzbasierte Präventionsmaßnahmen vernachlässigt.

- Einen Schaden für Mitarbeiter und Patienten bewirkt das Tragen von FFP2-Atemschutzmasken bei der normalen medizinischen Versorgung von InfluenzaFällen, denn solche Masken bieten hier keinen effektiveren Schutz als OP-Masken [7], sind aber erheblich teurer.

- Als patientenschädigend ist zurzeit auch die generelle räumliche Isolierung von MRSA-Patienten einzustufen, die für den betroffenen Patienten keinen Nutzen, wohl aber Schäden mit sich bringt [8]. Wirtschaftliche Schäden entstehen daneben durch die Isolierungskosten, während ein Nutzen selbst für die Mitpatienten nicht ausreichend belegt ist [9].

- Schäden für die Patienten entstehen durch die Installation kostspieliger OP-Lüftungsanlagen mit turbulenzarmer Verdrängungsströmung, deren infektionspräventiver Effekt als widerlegt gilt [10].

In der krankenhaushygienischen Realität ist vieles nicht so klar zu beurteilen wie in den oben genannten Beispielen und für viele Präventionsmaßnahmen existieren (noch) keine Kosteneffektivitätsanalysen. Ein reales Problem stellen aber auch Personen und Institutionen dar, die Maßnahmen entsprechend dem 4. Beispiel fordern (hohe Kosten bei widerlegter Wirksamkeit). Wer wider besseres Wissen solche Forderungen aufstellt, muss sich den Vorwurf gefallen lassen, nicht nur gegen den Grundsatz „primum prodesse“ zu verstoßen sondern sogar nach dem Grundsatz „primum nocere“ zu verfahren.

\section{Literatur}

1 Smith CM. Origin and uses of primum non nocere above all, do no harm! J Clin Pharmacol 2005; 45: $371-377$

2 Celis H, Fagard RH. White-coat hypertension: a clinical review. Eur J Intern Med 2004; 15: 348 - 357

3 Weihrauch TR, Gauler TC. Placebo - efficacy and adverse effects in controlled clinical trials. Arzneimittelforschung 1999; 49: 385-393

4 Barbuto JP, White Jr. GL, Porucznik CA, Holmes EB. Chronic pain: second, do no harm. Am J Phys Med Rehabil 2008; 87: 78-83

5 Body R, Foex B. On the philosophy of diagnosis: is doing more good than harm better than „primum non nocere“? Emerg Med J 2009; 26: 238-240

6 König HH, Riedel-Heller S. Prävention aus dem Blickwinkel der Gesundheitsökonomie. Internist 2008; 49: $146-153$

7 Loeb M, Dafoe N, Mahony J et al. Surgical mask vs N95 respirator for preventing influenza among health care workers: a randomized trial. JAMA 2009; 302: 1865-1871

8 Morgan DJ, Diekema DJ, Sepkowitz K, Perencevich EN. Adverse outcomes associated with Contact Precautions: a review of the literature. Am J Infect Control 2009; 37: 85-93

9 Korczak D, Schöffmann C. Medizinische Wirksamkeit und Kosten-Effektivität von Präventions- und Kontrollmaßnahmen gegen Methicillin-resistente Staphylococcus aureus (MRSA)-Infektionen im Krankenhaus. HTA-Bericht Bd. 100. Köln: Deutsche Agentur für Health Technology Assessment des Deutschen Instituts für Medizinische Dokumentation und Information www.dimdi.de, 2010

10 Kommission für Krankenhaushygiene und Infektionsprävention (KRINKO) beim Robert-Koch-Institut. Kommentar der KRINKO zur DIN 1946-4 (2008). Epidemiol Bulletin 2010; 4: 35 